\title{
EEG hyperscanning and behavioral synchronization during a joint action
}

\author{
Michela Balconi $^{1,2}$ - Maria Elide Vanutelli ${ }^{1,2}$ \\ ${ }^{1}$ Research Unit in Affective and Social Neuroscience, Catholic University of Milan, \\ Milan, Italy \\ ${ }^{2}$ Department of Psychology, Catholic University of Milan, Milan, Italy
}

DOI: http://dx.doi.org/10.7358/neur-2018-024-balc_michela.balconi@unicatt.it

\begin{abstract}
Brain-to-brain coupling during cooperation is a core question of study on social interactions. The aim of the present study was to investigate the neural basis of interbrain cooperation and the cognitive performance underlying the execution of jointactions by using EEG coherence measures. Synchronicity of the cognitive variables (response times, RTs, and error rates, ERs) in response to an attentional task, intersubjective coherence analysis on EEG frequency bands, and correlational measures between cognitive and brain activity were considered during some steps of progressive reinforcing conditions. Fifteen couples of subjects performed an attentional task in eight temporal steps, stressing their good performance at the end of each step. The induced feedback affected both the cognitive performance and brain-to-brain coupling by increasing behavioral and brain synchronization when a positive feedback was furnished to the participants for their performance. Secondly, about the cortical contribution, high coherence effect was mainly observed when a positive reinforce was produced, but only for some low frequency bands within the prefrontal left area, compared to the right one. Thus, also a left lateralization effect was reportable. Finally, the cognitive and EEG coherence measures were shown to be correlated, with a significant similar trend anchored to the progressive feedback.
\end{abstract}

Keywords: hyperscanning; joint-action; EEG; brain-to-brain coupling; social reinforce 


\section{INTRODUCTION}

Cooperative behavior is a model of social interaction that requires a coordinated actions between two or more inter-agents (Vanutelli, Nandrino, \& Balconi, 2016). In previous studies it was found that cooperative tasks often improve the subjective performance and they simultaneously contribute to positively modify the subjective perception of social role (Decety, Jackson, Sommerville, Chaminade, \& Meltzoff, 2004). It was supposed that this improving is due to intrinsic features of cooperative situations, since they may reinforce the sense of being-part of a whole, the sense of joint-performance, and the perceived self-efficacy in a dual task (Balconi \& Pagani, 2015; Chung, Yun, \& Jeong, 2015; X. Cui, Bryant, \& Reiss, 2013; Goldman, Stockbauer, \& McAuliffe, 1977). However in some cases it was also shown that cooperative conditions may reinforce the interpersonal cohesion at cost of a worse cognitive performance (Funane et al., 2011).

Moreover, a vast amount of evidence indicates that social interactions implicate a specific set of cortical regions which support the social behavior. Indeed, it was observed that a neural circuit including prefrontal, limbic, and striatal structures may reflect respectively the cognitive, emotional and behavioral components of social interactions during cooperation (Levitan, Hasey, \& Sloman, 2009). Such research also showed enhanced cortico-cortical communication and interconnections between these brain regions. In addition it was found that dorsal (DLPFC) and ventral (VLPFC) portions of the lateral prefrontal cortex are generally activated during social status inference (Balconi \& Bortolotti, 2012; Balconi \& Pagani, 2014, 2015; Chiao et al., 2009). The activation of DLPFC and VLPFC during social interactions may reflect the implication of brain regions which are related to the specific control over interaction processes, such as emotional responses to social actions, and to the regulation of socially appropriate behavior (Marsh, Blair, Jones, Soliman, \& Blair, 2009). Indeed, it was noted that these brain regions have been typically associated with the regulation of socioemotional responses and the behavioral inhibition system.

However, adopting an individual and not a brain-coupling perspective, many previous studies generally analyzed only single subjects at a time and their isolated performance in generic social tasks, since they did not include paired joint-actions or really interactive tasks. That is they are mostly measured in only one brain at a time by using various modalities, such as electroencephalography (EEG) (Cheung, Rutherford, Mayes, \& McPartland, 2010; Gao et al., 2009), positron-emission tomography (Fletcher et al., 1995; Gallagher, Jack, Roepstorff, \& Frith, 2002; Goel, Grafman, Sadato, \& Hallett, 1995), single photon-emission computed tomography (Baron-Cohen et al., 1994), functional magnetic resonance imaging (fMRI) (Fukui et al., 2006; Mitchell, Banaji, \& Macrae, 2005; Schippers, Roebroeck, Renken, Nanetti, \& Keysers, 2010; Stephens, Silbert, \& Hasson, 
2010), and functional near-infrared spectroscopy (fNIRS) (Balconi, Crivelli, \& Vanutelli, 2017; Balconi \& Vanutelli, 2016a,2016b; Grossmann \& Johnson, 2010; Grossmann, Oberecker, Koch, \& Friederici, 2010; Lloyd-Fox et al., 2009; Minagawa-Kawai et al., 2009; Suda et al., 2010). Consequently, little is known about how social functions are processed in interacting brains of more than one participant. In other cases research explored the asynchronous response (subsequential) by two or more subjects interacting each other, or they were just interested in comparing different social interactive conditions, such as competitive or cooperative tasks (Boone, De Brabander, \& van Witteloostuijn, 1999; Decety et al., 2004).

Since these series of studies did not use the simultaneous recording of the subjects involved in the experiment, but computed off-line measurements of synchronicity between the brain activities, further investigation is required to illustrate the contribution of the neural structures in two-individuals when subjects cooperate toward a common goal during a joint-action. In addition, it is extremely useful to include a real dynamic interaction where the co-partners are actively implicated in the cooperative exchange. At this regard, the hyperscanning approach introduces an innovative perspective to comprehend the synchronized strategy of two interacting brains (Holper, Scholkmann, \& Wolf, 2012). Indeed, it is reasonable to suppose that the relationship between spatial brain-activity patterns of two people is effective in the investigation of brain functions related to social interactions. For example, research of interpersonal neural synchronization using functional near-infrared spectroscopy (fNIRS) in hyperscanning paradigms revealed this synchrony in cooperative verbal communication (Nozawa et al., 2013). Synchrony was also observed in the dorsomedial prefrontal cortex (DMPFC) during cooperative interaction. These additional findings suggest that DMPFC may be particularly engaged when theory-of-mind (ToM) is required for cooperative social interactions.

However, with respect to fNIRS hyperscanning, EEG hyperscanning studies have higher temporal resolution and could be used to capture moment-to-moment interactions. This method has the potential to significantly extend fNIRS applications to social cognitive research (Liu et al., 2016). At present limited research applied this measure to cooperative dynamics. Some previous studies examined the relationship of brain rhythm synchronization during speech between subjects (Kawasaki et al., 2013), but also during music playing (Lindenberger, Li, Gruber, \& Müller, 2009; Sänger, Müller, \& Lindenberger, 2012). Other studies, instead, explored motor synchronization with rythmic finger movements (Konvalinka et al., 2014; Naeem, Prasad, Watson, \& Kelso, 2012; Tognoli, Lagarde, DeGuzman, \& Kelso, 2007), but also the processes underlying decisionmaking (Yun, Watanabe, \& Shimojo, 2012).

In addition, it should be noted that the EEG hyperscanning acquisition was 
used in response to cognitive performance without a specific external feedback which may reinforce the effect of the joint-action (F. Cui, Zhu, Duan, \& Luo, 2015; Dommer, Jäger, Scholkmann, Wolf, \& Holper, 2012; Saito et al., 2010). Therefore, the present study aimed at moving towards a two-person hyperscanning by investigating the EEG correlates of between-brain synchrony during a joint task in presence of an external feedback related to the interpersonal joint performance. Thus, we created an explicit and strongly reinforced social dynamic based on incidental skills in the context of a dyadic interaction. In contrast with previous studies (Zink et al., 2008), we included a more ecologic task, where subjects were required to constantly compare their performance with that of the other subject. Indeed the subject's performance was artificially manipulated in a dyadic vis-à-vis cooperative condition which stressed the joint-effect of a coordinated strategy (a better cognitive performance). This comparison allowed to test the effect of the dynamic modifications on subject's brain activity related to the second subject's brain activity.

Therefore, the aim of the present study was to investigate the neural basis of inter-brain cooperation and the cognitive performance underlying the execution of joint-actions by using EEG coherence measure. Based on our hypotheses, firstly direct interactions between the two brains and the observed external feedback of their performance (increased performance during the task) may effectively impact on the social and cognitive success with a better cognitive performance and an inter-brain synchronization. Secondly, the brain correlates of this social competencies are supposed to involve the prefrontal cortex, with a specific role of the DLPFC, since this area may impact on the interpersonal performance in dyadic interactive contexts. Finally, we expected this increased cognitive performance will be related to the increased inter-brain synchronization during the task, with significant higher brain-coupling. The two measures (behavioral synchronicity and inter-brain coherence) are expected to be correlated each-other during the task.

\section{METHOD}

\subsection{Subjects}

Fifteen couples of subjects (thirty-two total subjects) were recruited. They were undergraduate students $(M=23.21, S D=2.12)$. Each couple was composed by two individuals of the same sex matched for age. They did not meet and were not familiar before the experimental session. The participants were all right-handed, they presented normal or corrected-to-normal visual acuity, and gave informed written consent to participate in the study. Exclusion criteria 
were history of psychopathology (Beck Depression Inventory, BDI-II, Beck et al., 1996; State-Trait-Anxiety-Inventory (STAI, Spielberger et al., 1970) for the subjects and immediate family. No neurological or psychiatric pathologies were observed. The research was approved by the local ethics committee of the Department of Psychology, Catholic University of Milan. No payment was provided for subjects' performance.

\subsection{Procedure}

Subjects were comfortably seated in a moderately darkened room with a computer screen located approximately $60 \mathrm{~cm}$ from their eyes. They were required to perform a simple task for sustained selective attention (modified from the original task of Balconi and Pagani, 2014). To engage subjects in the task, they were told that some cognitive attentional measures were used to evaluate their subjective skills and that these measures are usually used as screening to test future professional career success and teamwork capabilities. In addition, the cooperative and joint nature of the task was stressed by telling subjects that their scorings were based on the capacity to synchronize their responses, in term of accuracy (error rates, ERs) and response times (RTs), with the other subject. Thus, the development of a joint cooperative strategy by the couple was reinforced. They were seated side-by-side, separated by a dark screen to prevent visual contact and to avoid any effect due to the nonverbal behavior.

The attentional task required to select target stimuli between non-targets, based on four different combinations of shape and color: triangles and circles, blue and green. Each target was displayed on the screen and subjects were asked to memorize it. Then, stimuli were presented one after another. The target stimulus features were varied in every experimental block, composed by 25 trials. Subjects were instructed to answer all the stimuli by pressing left/right buttons to decide for targets or non targets. Each stimulus remained on the screen for 500 msec, with a $300 \mathrm{msec}$ inter-stimulus interval (ISI). Each trial was composed by three stimuli. After each trial subjects received a feedback in the form of two uparrows (high cooperation score); a dash (mean performance); or two down-arrows (low cooperation score). This feedback was shown for $5000 \mathrm{msec}$ and then an inter-trial interval (ITI) occurred for other $5000 \mathrm{msec}$. The task was composed by eight general sessions (eight Block of 25 trials each), and each session received a general feedback of the joined performance at the end (Figure 1). Thus participants constantly received a general evaluation of their cooperative performance: both trial-feedbacks (every three trials) and the general-feedback were fixed and after each block subjects were told they had a good cooperation (temporal synchronicity and paired performance: score with $87 \%$ in terms of speed, and $92 \%$ in terms of accuracy). They were also encouraged to maintain

Neuropsychological Trends - 24/2018

http://www.ledonline.it/neuropsychologicaltrends/ 
their performance level during the experiment. During the task, after an initial mean performance, subjects were constantly reinforced about their good cooperation by presenting the up-arrows in $70 \%$ of cases, while the dash or the down-arrows appeared in 30\% of cases. In addition after each block of 25 trials, subjects were required to evaluate their performance and efficacy in term of their ranking on a seven-point Likert scale (from one $=$ most decreased ranking due to performance, to seven $=$ most improved ranking due to performance). Participants were strongly engaged in the hierarchical context (93\% told to be strongly engaged, as reported in a post-experimental questionnaire). Subjects were also required to self-report their degree of trust of the exact feedback of the performance, which showed high trust $(95 \%)$, a relevance of the task for social status representation $(96 \%)$, the perceived improved ranking position during the task (94\%).

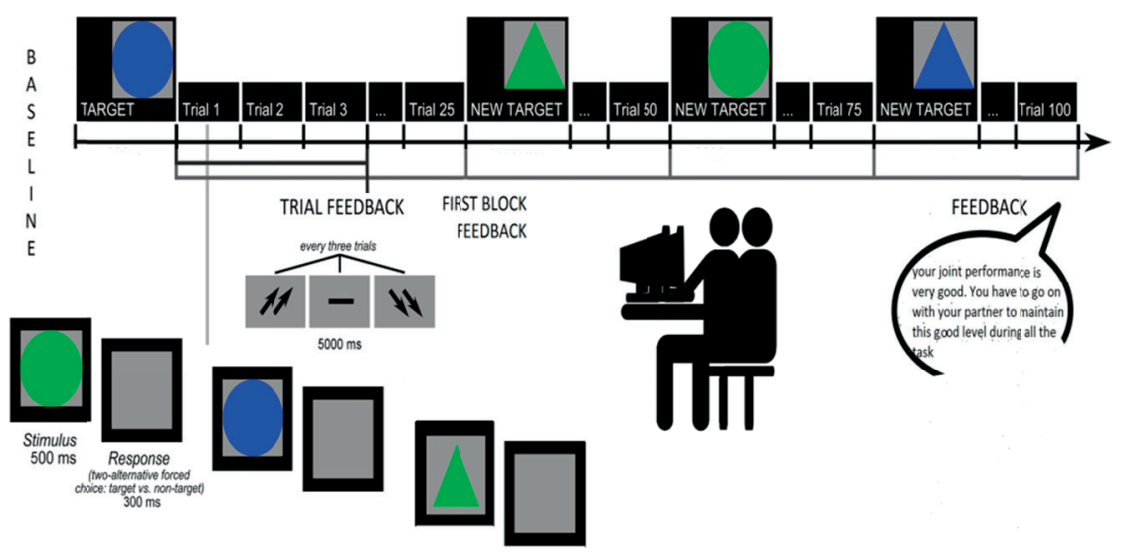

Figure 1. Experimental procedure which represents setting and the joint task

\subsection{EEG recording and analysis}

EEG recordings were performed with two 16-channel EEG-systems (V'-AMP: Brain Products, München. Truscan: Deymed Diagnostic, Hronov). ElectroCaps with $\mathrm{Ag} / \mathrm{AgCl}$ electrodes were used to record EEGs from active scalp sites referred to the earlobes (10/5 International System; Oostenveld and Praamstra, 2001). Data were acquired using a $1000 \mathrm{~Hz}$ sampling rate, with a $0.01-100 \mathrm{~Hz}$ input filter. An off-line common average reference was 
successively computed to reduce problems associated with the signal-to-noise ratio (Ludwig et al., 2009). EOG electrodes were placed at the outer canthi to detect eye movements. The impedance of the recording electrodes was monitored for each subject prior to data collection and was always kept below 5 $\mathrm{k} \Omega$. After performing EOG correction and visual inspection, only artefact-free trials were considered (rejected epochs, 2\%). The signal was visually scored, and portion of the data that contained artefacts were removed to increase specificity. Blinks were also visually monitored. Ocular artefacts (eye movements and blinks) were corrected using an eye-movement correction algorithm that employs a regression analysis in combination with artefact averaging (Sapolsky, 2004). EEG activity was recorded on positions AFF1h, AFF2h, Fz, FFC3h, FFC4h, C3, C4, Cz, P3, P4, Pz, T7, T8, O1, O2.

The digital EEG data were bandpass filtered offline $(0.1-40 \mathrm{~Hz}, 48$ $\mathrm{dB} /$ octave rolloff) and frequency power data were computed by FFT transformation for standard frequency bands: delta $(0.5-4 \mathrm{~Hz})$, theta $(4-8 \mathrm{~Hz})$, alpha $(8-12 \mathrm{~Hz})$, beta $(14-20 \mathrm{~Hz})$. An individual average power value for each experimental condition and for baseline recordings was calculated for each EEG channel. For the statistical analysis, AFF1h-AFF2h FFC3h-FCC4h C3C4 T7-T8 P3-P4 channels were considered for each frequency band.

Inter-channel coherence analysis was applied to each pair of subjects for each channel. EEG coherence was computed by the Fast Fourier Transformation method for each band. Successively EEG coherence was computed by a correlation analysis, as the spectral cross-correlation between two electrodes normalized by their power spectra for each pair of subject. Its values range from 0 to 1 and indicate the amount of covariation between recording localizations on the scalp (Balconi, Brambilla, \& Falbo, 2009; Balconi \& Mazza, 2009).

\section{RESULTS}

\subsection{Statistical analysis}

The RTs (msec) were recorded from the stimulus onset, and ERs were calculated as the total number of incorrect detections out of the total trial for each category. Therefore higher values represented increased incorrect responses.

Three orders of analyses were performed with respect to behavioral (ER; RTs) and EEG measures. A first order of analysis (repeated measures ANOVAs) was finalized to test the performance of the subjects taking into account the behavioral measures (ERs and RTs) in response to the feedback. The second 
order of analysis calculated the synchronization indices within each couple (correlational and coherence analysis respectively for behavioral and EEG measures). It included a Pearson correlation coefficient within each couple for each block of the task (8) for ERs and RTs. In the case of EEG, coherence analysis was applied to the data for each band and channels within each couple of subjects.

Based on these indices the third order of analysis was finalized to test the statistical significance of these behavioral synchronization and EEG coherence by using repeated measure ANOVA. ANOVAs were applied distinctly to ERs and RTs Pearson coefficient values with independent factor Block (8). For the EEG, ANOVAs included factors Block (8) $\mathrm{x}$ Lateralization $\mathrm{x}$ Localization applied to the coherence values.

Finally, in order to test the correlation between the cortical coherence measures for the EEG and the correlational values for the cognitive performance (ERs and RTs) a correlational analysis was applied between Pearson coefficients and coherence values for each region of interest (ROI) within each band.

For all the ANOVA tests, the degrees of freedom have been corrected using Greenhouse-Geisser epsilon where appropriate. Post-hoc comparisons (contrast analyses) were applied to the data. Bonferroni test was applied for multiple comparisons.

\subsection{ER and RTs}

\subsubsection{Performance}

For ERs measure, ANOVA indicated significant effect for Block $(F[7,28]=6.98$, $p \leq .001, \eta^{2}=.33$ ) (figure 2a). As shown by post-hoc comparisons (contrast analysis for paired comparisons) ERs decreased across the blocks. Indeed significant effects were found between the first block and the following second $\left(F[7,28]=7.54, p \leq .001, \eta^{2}=.34\right)$ and third block $\left(F[7,29]=8.05, p \leq .001, \eta^{2}\right.$ $=.37$ ), with lower ERs for the second/third. Finally the last two blocks differed from all the previous blocks, with lower ERs compared to the other values (for all comparisons $p \leq .001$ ).

About RTs, ANOVA indicated significant main effect for Block $(F[7,29]=$ 9.05, $p \leq .001, \eta^{2}=.39$ ), with decreased RTs across the blocks (figure $2 \mathrm{~b}$ ). In fact, the first three blocks differed from the fourth, fifth and sixth blocks, with lower RTs (for all comparisons $p \leq .001$ ). Similarly, the last two blocks differed from the previous ones, with lower values compared to the other blocks (for all comparisons $p \leq .001)$. 


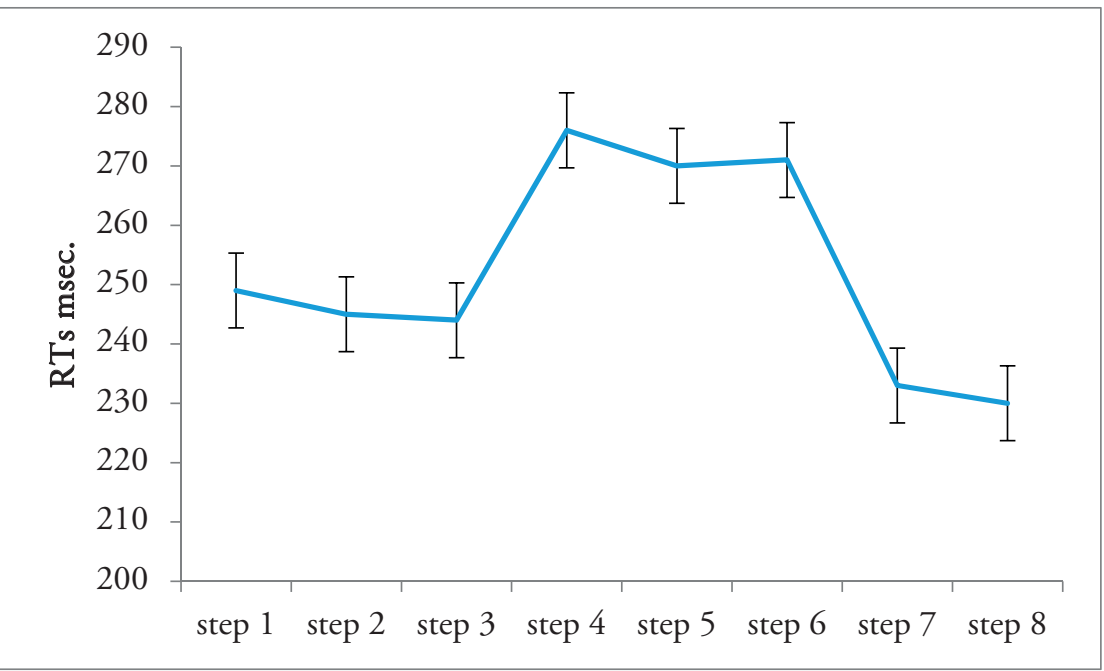

Figure 2a. ERs/RTs modulation as a function of the eight blocks. Decreased ERs were found for second-third block and the last two blocks

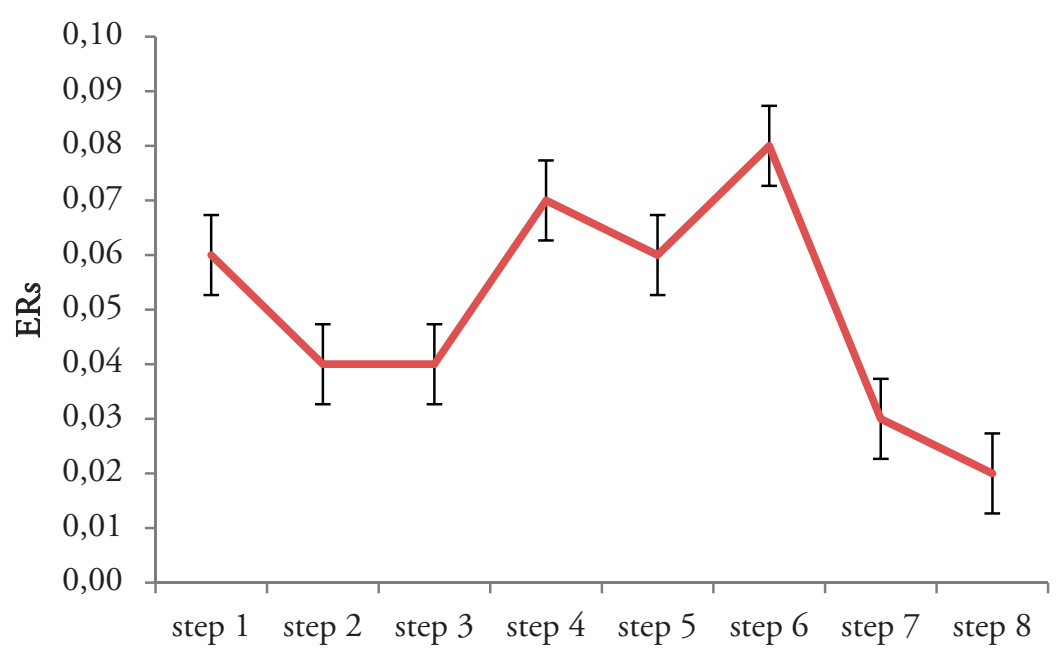

Figure 2b. ERs/RTs modulation as a function of the eight blocks. Decreased RTs were found for first three and the last two blocks 


\subsubsection{Correlational measures}

For ERs measures, ANOVA indicated significant effect for Block $(F[7,14]=$ $6.90, p \leq .001, \eta^{2}=.34$ ) (figure 3a). As shown by post-hoc comparisons (contrast analysis for paired comparisons) ERs coefficients increased across the blocks for the couples. Indeed significant effects were found between the first block and the second $\left(F[7,14]=8.02, p \leq .001, \eta^{2}=.37\right)$ and third $(F[7,14]=8.89, p \leq .001$, $\eta^{2}=.38$ ) block, with higher coefficients for the second/third block. Finally the last two blocks differed from all the previous blocks, with the higher values of ERs coefficients compared to the other values (for all comparisons $p \leq .001$ ).

About RTs, ANOVA indicated significant main effect for Block $(F[7,14]=7.16$, $p \leq .001, \eta^{2}=.35$ ), with increased RTs coefficients across the blocks (figure $3 \mathrm{~b}$ ). In fact, the first three blocks differed from the fourth, fifth and sixth blocks (for all comparisons $p \leq .001)$. Similarly the last two blocks differed from the previous ones, with higher coefficient values compared to the other blocks (for all comparisons $p \leq .001)$.

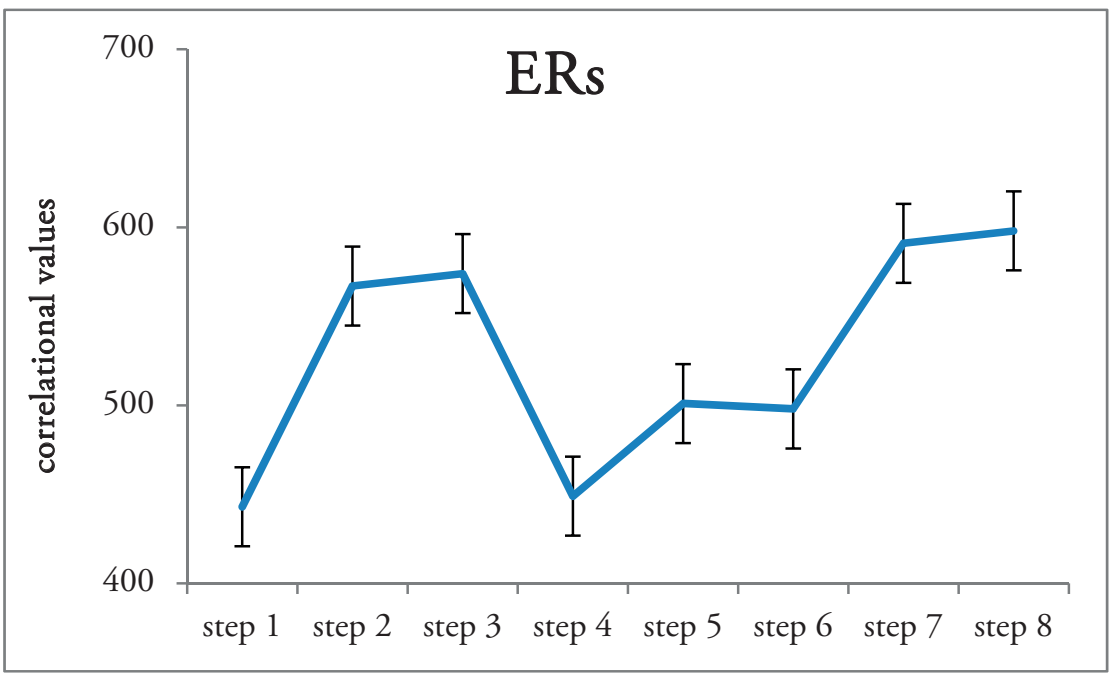

Figure 3a. Mean correlational values (intra couple) for ERs/RTs. ERs coefficients increased for the secondlthird and the last two blocks 


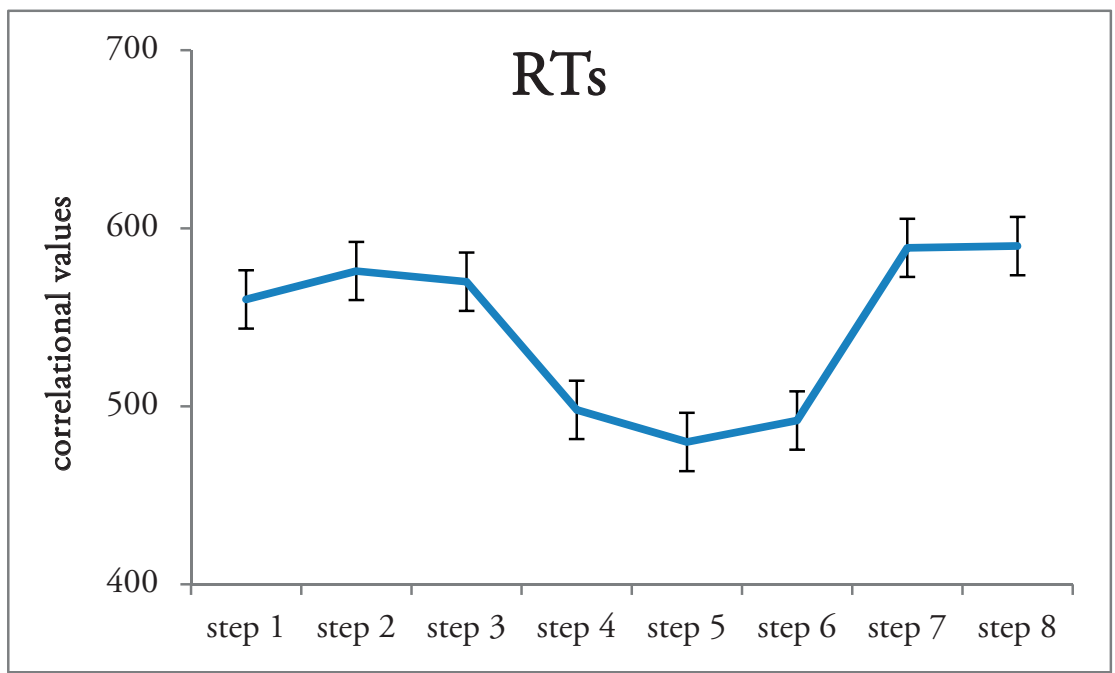

Figure 36. Mean correlational values (intra couple) for ERs/RTs. RTs coefficients increased for the first three and the last two blocks

\subsubsection{ANOVAs on the coherence measures}

A series of ANOVA were applied to the dependent coherence values calculated for each frequency band, with independent factors Block (8), Localization (4) and Lateralization (2).

Localization (four sites: frontal, temporo-central, parietal) and Lateralization (two left, and right) were calculated. Specifically, we measured left and right frontal (AFF1h, AFF2h, FFC3h, FFC4h), temporo-central (C3, C4, T7, T8), and parietal (P3, P4) brain activity.

For delta, repeated measure ANOVA showed significant effect for Block ( $F[7$, $\left.14]=6.92, p \leq .001, \eta^{2}=.32\right)$ and Block $\mathrm{x} \operatorname{Loc} \mathrm{x}$ Lat $\left(F[21,42]=7.52, p \leq .001, \eta^{2}\right.$ $=.34$ ) (figure 4a). Indeed, increased coefficients were found from the third block compared with the first two (respectively $F[7,14]=7.12, p \leq .001, \eta^{2}=.33 ; F[7$, $14]=7.02, p \leq .001, \eta^{2}=.33$ ). Similarly, the last two blocks showed a significant increased coefficient compared to the previous ones (for all comparisons $p \leq .001$ ). Secondly, about the interaction effect, the frontal left hemisphere showed higher coefficient values compared to the right hemisphere for the fourth, fifth, sixth, seventh and eighth block (for all comparisons $p \leq .001$ ). 
For theta, repeated measure ANOVA showed significant effect for Block ( $F[7$, $\left.14]=8.12, p \leq .001, \eta^{2}=.36\right)$ and Block $\mathrm{x}$ Loc $\mathrm{x}$ Lat $\left(F[7,14]=7.74, p \leq .001, \eta^{2}\right.$ $=.34$ ) (figure $4 \mathrm{~b}$ ). As shown, increased coefficients were found from the third block compared with the first two blocks $\left(F[7,14]=7.52, p \leq .001, \eta^{2}=.34 ; F[7\right.$, $\left.14]=6.93, p \leq .001, \eta^{2}=.31\right)$. In addition, the last two blocks showed a significant increased coefficient compared to the previous ones (for all comparisons $p \leq .001$ ). Secondly, about the interaction effect, the frontal left hemisphere showed higher coefficient values compared to the right hemisphere for the fifth, sixth, seventh and eighth block (for all comparisons $p \leq .001$ ).

For alpha, significant effects were found for Blocks $(F[7,14]=7.84, p \leq$ $.001, \eta^{2}=.35$ ), with increased coefficients from the fourth block compared with the all the previous blocks (all comparisons $p \leq .001$ ) (figure 4c). No other effect was statistically significant.

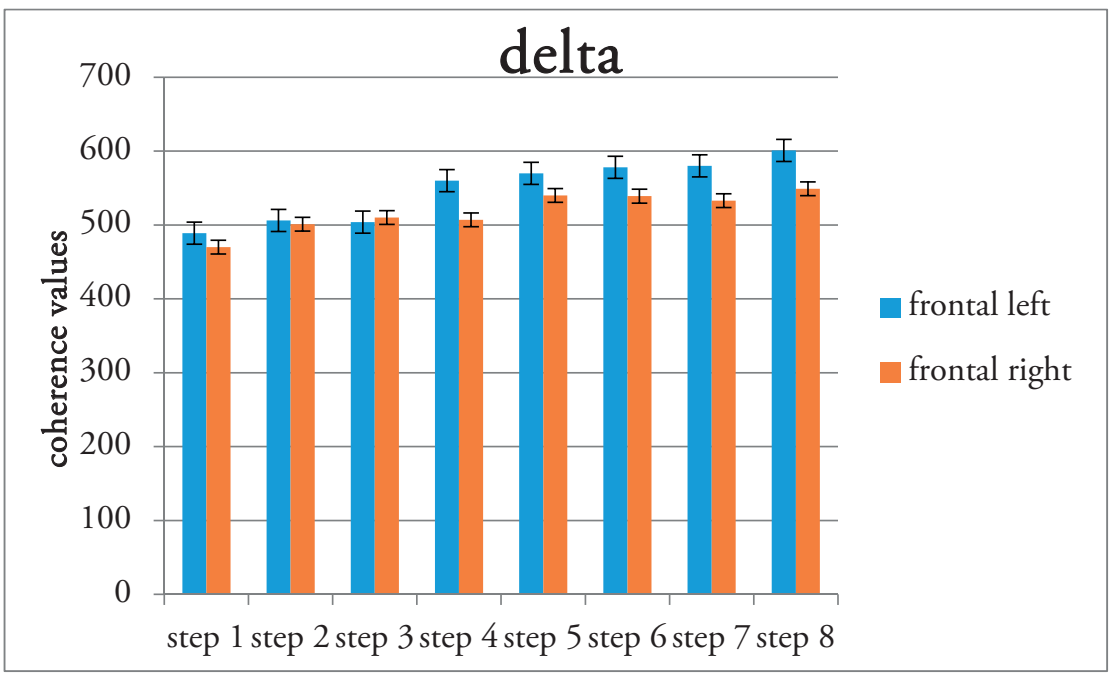

Figure 4a. Mean coherence measures (intra-couple) for the frequency bands as a function block and cortical area. Coherence measure modulation for delta for frontal leftright area and the eight blocks 


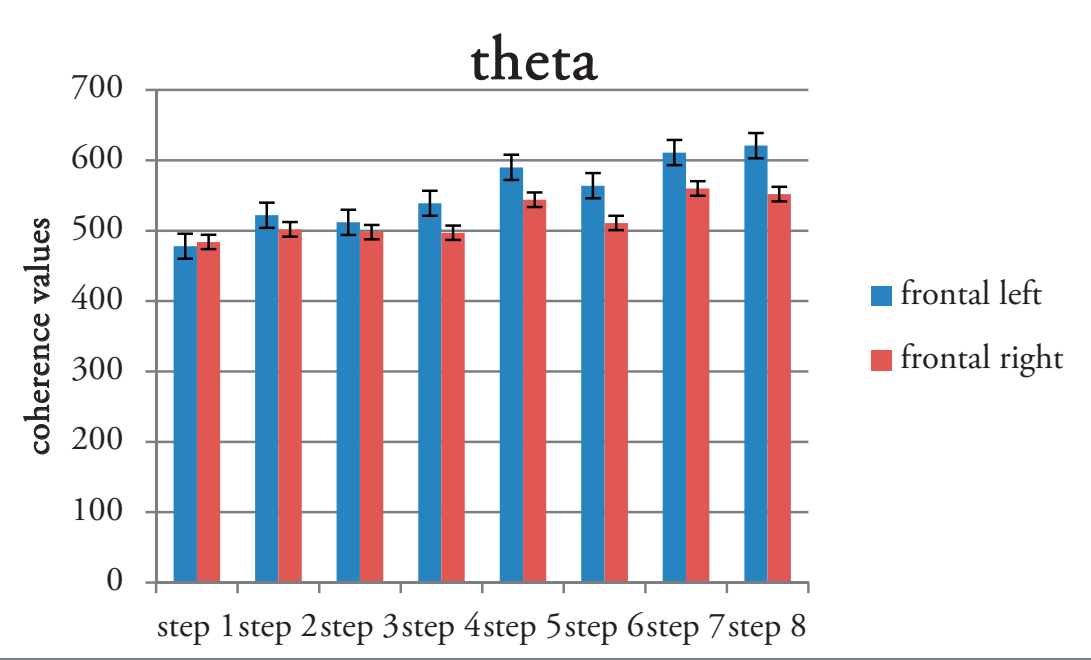

Figure 4b. Mean coherence measures (intra-couple) for the frequency bands as a function block and cortical area. Coherence measure modulation for theta for frontal leftright area and the eight blocks

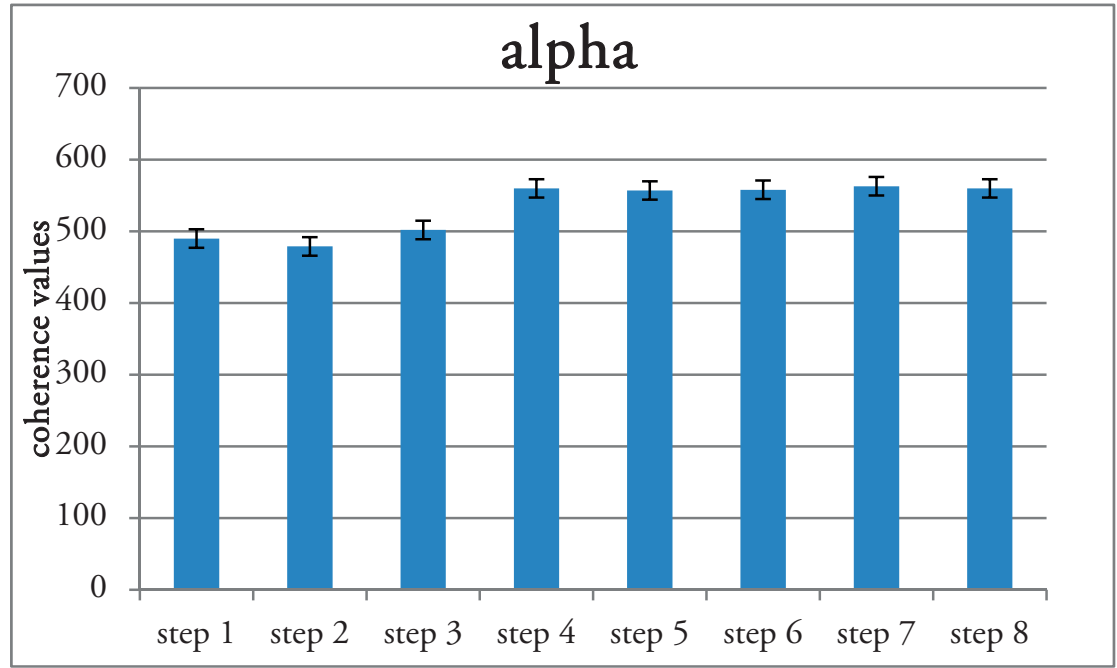

Figure 4c. Mean coherence measures (intra-couple) for the frequency bands as a function block and cortical area. Coherence measure modulation for alpha for the eight block 


\subsubsection{Correlational values behaviorallcoherence measures}

Pearson correlational values were calculated between ERs and RTs coefficients and the coherence values for each frequency band within specific regions of interests (ROIs, left/right frontal).

Significant correlations were found between left delta activity and RTs measures for all the blocks from the fourth block (respectively $\mathrm{r}^{2}=.485, p \leq .001$ $\left.\mathrm{r}^{2}=.534, p \leq .001 \mathrm{r}^{2}=.592, p \leq .001 \mathrm{r}^{2}=.599, p \leq .001 \mathrm{r}^{2}=.555, p \leq .001\right)$ (figure 5a).

Similarly, significant correlations were found between left theta and RTs for all the blocks from the fourth block (respectively $\mathrm{r}^{2}=.616, p \leq .001 \mathrm{r}^{2}=.570, p \leq$ $.001 \mathrm{r}^{2}=.555, p \leq .001 \mathrm{r}^{2}=.598, p \leq .001 \mathrm{r}^{2}=.545, p \leq .001$ ) (figure 5b).

In contrast, alpha and beta did not show significant relations.

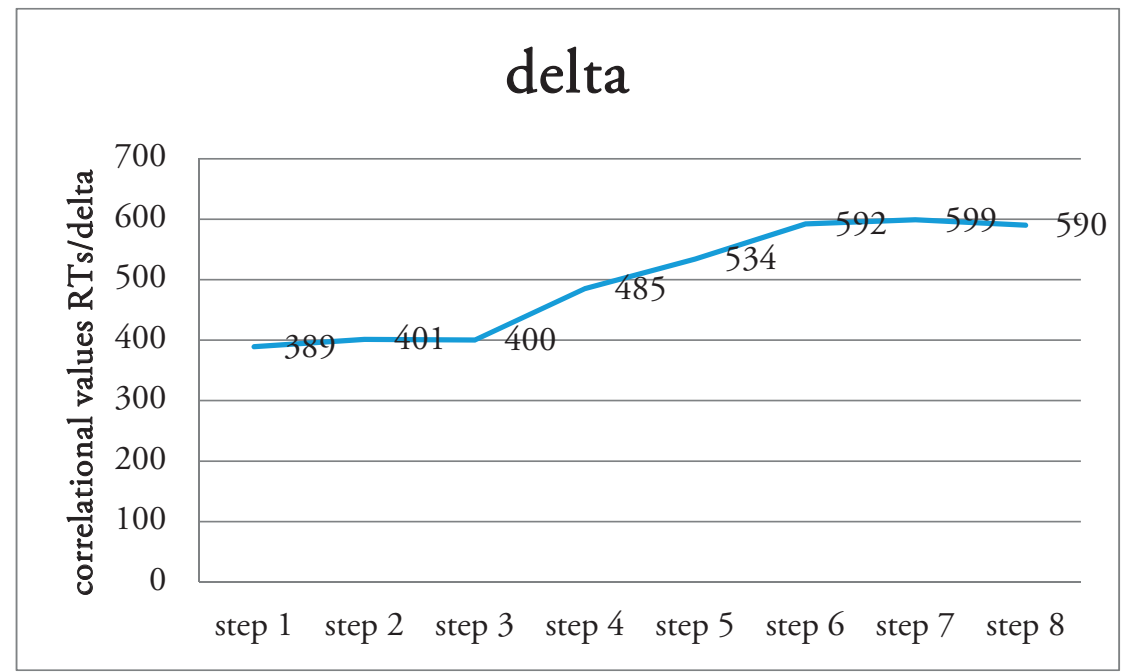

Figure 5a. Mean correlational values between RTs coefficients (intra-couple) and frequency band coherence (intra-couple) values as a function of block. RTs-delta correlations 


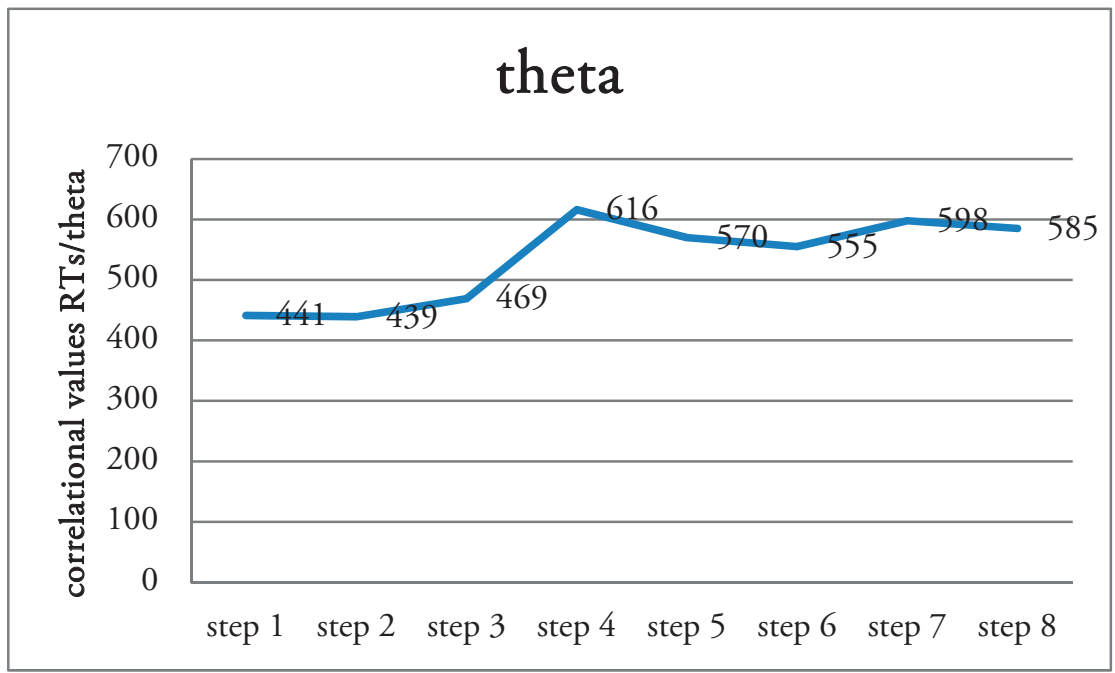

Figure 5b. Mean correlational values between RTs coefficients (intra-couple) and frequency band coherence (intra-couple) values as a function of block. RTs-theta correlations

\section{DISCUSSION}

By using an EEG hyperscanning paradigm, the present research analyzed a joint-action focusing on the cognitive performance and the brain activity (brain-to-brain coupling) during a cooperative task which reinforced the positive outcomes of the inter-subjective action. Synchronicity of the cognitive variables (RTs and ERs) in response to an attentional task, coherence analysis on EEG frequency bands for inter-brain coupling, and correlational measures between cognitive and brain activity were considered during the steps of progressive reinforcing conditions. Based on our results, we may highlight some main effects. A first result was related to the systematic feedback effect on both the cognitive performance and brain-to-brain coupling. Indeed the external feedback induced a gradually increased behavioral and brain synchronization when a positive reinforce (positive feedback) was furnished to the participants for their performance. Secondly, about the cortical contribution, this coherence effect was mainly observed for some specific frequency bands (low frequency) and selected brain areas, that is the prefrontal left area compared to the right 
one. Thus, also a left lateralization effect was reportable. Finally, the cognitive and EEG coherence measures were shown to be correlated each other, with a significant similar trend anchored to the progressive feedback during the task.

\section{Behavioral synchronization within the couple}

The first main effect we observed was related to a process of gradual synchronization with a significant initial increasing in the joint cognitive performance, a successive plateau effect, followed by a new increased jointaction for both ERs and RTs. This modulation may underline a strategic trend, with significant initial necessity to synchronize (mainly the second and third step), and a successive plateau effect within the central steps. A successive higher synchronization at the end of the task, the highest among the other previous steps, elucidated a sort of optimization of the cognitive performance within each couple.

It should be highlighted that the cognitive synchronization within the couple for both RTs and ERs was accompanied by an increased performance during the task. That is, as shown by ANOVAs, across the task the subjects' general performance improved with a significant better effect (reduced ERs and $\mathrm{RTs}$ ) especially for the last phase of the task. A sort of familiarization and cognitive learning may be adduced to explain this trend. In addition, also the presence of a social reinforce (represented by a better joint-action) may have acted as an intrinsic stimulation to produce a more efficient response to the cognitive task in order to reach the optimal degree at the end of the task.

This was partially in contrast with previous results that pointed out the cost of cooperation compared with competition, which generally induces a significant gaining effect (reduced RTs) due to the focus allocated more on the individual proficiency than to interpersonal goals (Tauer \& Harackiewicz, 2004). In contrast, we observed a general optimization effect for the interpersonal performance when a cooperative task was provided. This fact should be due to the reason that our task highly stressed the joint-action and the relevance to produce a proficient interpersonal outcomes.

\section{Inter-brain coherence: the social cooperative brain-to-brain coupling effect}

About the inter-brain coupling, we observed a consistent and relevant increased shared brain activation for the couple of participants, mainly in concomitance to an increasing positive social feedback. That is, this homologous inter-brain activity emerged gradually across the performance for most of the couples. Therefore we may state that the externally induced reinforcing condition acted on the joint cortical responsiveness and that the feedback effect was efficient on 
both the cognitive performance and brain-to brain coupling. In fact, higher coherence was found for the last part of the task as for the cognitive variables, thus underlining the impact of the external increasing positive feedback on both the electrocortical and cognitive level. That is, we may suppose that the external reinforce may have modulated the effective joint-behavior inside the couple, with relevant convergence of the increased cognitive performance and brain activity by the two inter-agents. In other terms, we may suggest that the two levels, behavioral and cortical, were effective in signaling the social effect of the reinforcing feedback, from one hand; that they share a similar responsiveness to external conditions which stress the joint significance of intersubjective actions, from the other hand.

It should be noted that this effect of brain activity synchronization was mainly related to some specific frequency bands. Indeed we found a more relevant implication of both delta and theta low frequency bands than the higher frequency bands (alpha and beta). As previously shown, delta and theta may represent a significant marker of the social competencies, empathy and emotional engagement.

Indeed, for what concerns theta band, it has been shown that increased frontal activity has been associated to strategic control and conflict monitoring in social contexts (Billeke, Zamorano, Cosmelli, \& Aboitiz, 2013; Cristofori et al., 2013). For example, Kawasaki and colleagues (Kawasaki, Yamada, Ushiku, Miyauchi, \& Yamaguchi, 2013) found that the behavioral synchronization of speech rhythms was correlated with the theta/alpha oscillatory amplitudes, which were enhanced and synchronized between two subjects. Interestingly, this effect wasn't present during the human-machine alternating speech task. Also, increased theta activity was found during empathic processes, such as empathy for pain (Mu, Fan, Mao, \& Han, 2008).

Considering delta band, Knyazev and colleagues showed that theta and delta synchronization is stronger during the presentation of emotional than neutral stimuli, and in those subjects who are more sensitive, or experience higher emotional involvement (Balconi, Grippa, \& Vanutelli, 2015; Knyazev, Slobodskoj-Plusnin, \& Bocharov, 2009; Paré, 2003). In addition, both delta and theta modulations were found to be related to arousing power of stimuli. Therefore, theta and delta may be responsive to motivational and attentional significance of relevant emotional cues or context per se (Balconi et al., 2009; Balconi \& Pozzoli, 2009; Başar, 1999).

It should also be underlined that some specific brain areas were highly implicated in this brain coupling effect for low-frequency bands in the form of a prefrontal contribution. Previous results revealed that prefrontal areas are prominent in social status regulation and joint actions (Haruno \& Kawato, 2009; Karafin, Tranel, \& Adolphs, 2004; Suzuki, Niki, Fujisaki, \& Akiyama, 2011). Also, using 
EEG-based hyperscanning technique, specific DLPFC activation emerged during reciprocal interactions (De Vico Fallani et al., 2010). In the present research we observed a similar effect, with significant increased PFC activity in response to positively reinforced joint action during the cognitive task. This prefrontal brain area was supposed to have an evolutionary relevance in social perception, especially when the interpersonal exchanges are crucial for the maintenance of social relationships. Therefore it is plausible to suppose that this area has specialized mechanisms to perceive social status and joint-actions performance. Specifically, prior work has suggested a main role of the PFC (mainly the VMPFC) in responding to status (Karafin et al., 2004). Recent studies investigating the effect of partner strategies found differential activation in the DLPFC when playing with cooperative, neutral, and non-cooperative human partners (Suzuki et al., 2011; Weiland, Hewig, Hecht, Mussel, \& Miltner, 2012) and activation in the superior temporal sulcus during successful adaption to the strategies of computer agents (Haruno \& Kawato, 2009). Additionally, De Vico Fallani and colleagues using the EEG hyperscanning technique have reported activation in this region during reciprocal interaction in iterated Prisoner's Dilemma games (De Vico Fallani et al., 2010).

However it should be taken into consideration that, in this study, the left hemisphere activity was demonstrated to be mainly prominent to explain our results. As pointed out by Koslov and colleagues (Koslow, Mendes, Pajtas, \& Pizzagalli, 2013), correlational research suggests that frontal cortical asymmetry in favor of the left hemisphere is associated with approach motivation, with the ability to regulate negative emotions, and with general wellbeing (Balconi \& Mazza, 2010; Davidson, 1993; Harmon-Jones, Gable, \& Peterson, 2010; Jackson et al., 2003; Urry et al., 2004). Starting from this evidence, they explored resting intracortical activity during social threat and found that participants with higher resting activity in the left vs. right DLPFC cortex exhibited more adaptive, approach-oriented cardiovascular stress responses. In addition, recent research revealed the significance of the left DLPFC for the empathic behavior and the emotional attuning during interpersonal actions (Balconi et al., 2015; Balconi \& Vanutelli, 2015; Vanutelli \& Balconi, 2015).

\section{To summarize: behavioral and brain synchronicity in cooperation}

More generally, we observed a high-level joint trend for both cortical and behavioral response to cooperative contexts. Mainly this similar trend was observable for brain and behavioral correlates within the second part of the task (higher correlation for the last blocks more than the first blocks). It is interesting to note that this "improved coupled brain effect" was also accompanied by a significant increased cognitive performance (decreased ERs and RTs). Indeed it was found that subjects highly improved their cognitive outcomes in response to the external reinforce. 


\section{CONCLUSIONS}

To summarize the cortical and behavioral data showed to be matched, with a similar trend of paired responsiveness for both cognitive behavior and cortical activity, which underlined the main effect of the artificially induced positive social reinforce on the inter-subjective joint performance.

However it should be noted a temporal delayed effect between behavioral and brain synchronization. That is, in comparison with the cognitive performance, we have to underline a delayed effect for the achievement of interbrain coherence related to the cortical brain oscillations. Indeed the optimization of the brain coupling was found from the fourth interval to the end, whereas the behavioral synchronicity within the couple was obtained before (from the second time interval to the successive). Two interpretations may be suggested for this temporal delay: the goal to reach the joint-performance has an immediate and short time effect on the behavioral level, with a successive concatenated effect on the brain level (increased brain coherence coefficients); in contrast they may be represented as two independent levels and the temporal delay between them is due to the relative different time these levels need to produce their visible effect respectively in term of correlation and coherence values.

Some limitations should be reported for the present study. Firstly the limited number of effective couples: this number should be improved in future research. Secondly, to better evaluate the cortical localization of the brain coupling effect, a higher density registration of EEG should be provided. Thirdly a different task (more focused on social than cognitive performance) should be used to analyze a more ecological social condition where cooperation could be tested. Finally some intra-personal features (such as personality empathic trait or proactive behavior like Behavioral Activation System - BAS - construct) should be included to verify the contribution of subjective attitudes in cooperative hyperlink conditions.

\section{REFERENCES}

Balconi, M., Bortolotti, A. (2012). Resonance mechanism in empathic behavior. BEES, BIS/BAS and psychophysiological contribution. Physiology and Behavior, 105(2), 298-304.

Balconi, M., Brambilla, E., \& Falbo, L. (2009). BIS/BAS, cortical oscillations and coherence in response to emotional cues. Brain Research Bulletin, 80(3), 151-157. 
Balconi, M., Grippa, E., \& Vanutelli, M. E. (2015). What hemodynamic (fNIRS), electrophysiological (EEG) and autonomic integrated measures can tell us about emotional processing. Brain and Cognition, 95, 67-76.

Balconi, M., \& Mazza, G. (2009). Brain oscillations and BIS/BAS (behavioral inhibition/activation system) effects on processing masked emotional cues. ERS/ERD and coherence measures of alpha band. International Journal of Psychophysiology, 74(2), 158-165.

Balconi, M., \& Mazza, G. (2010). Lateralisation effect in comprehension of emotional facial expression: a comparison between EEG alpha band power and behavioural inhibition (BIS) and activation (BAS) systems. Laterality, 15(3), 361-384.

Balconi, M., \& Pagani, S. (2014). Personality correlates (BAS-BIS), self-perception of social ranking, and cortical (alpha frequency band) modulation in peergroup comparison. Physiology \& Behavior, 133C, 207-215.

Balconi, M., \& Pagani, S. (2015). Social hierarchies and emotions: cortical prefrontal activity, facial feedback (EMG), and cognitive performance in a dynamic interaction. Social Neuroscience, 10(2), 166-178.

Balconi, M., \& Pozzoli, U. (2009). Arousal effect on emotional face comprehension. Frequency band changes in different time intervals. Physiology and Behavior, 97(3-4), 455-462.

Balconi, M., \& Vanutelli, M. E. (2015). Emotions and BIS/BAS components affect brain activity (ERPs and fNIRS) in observing intra-species and inter-species interactions. Brain Imaging and Behavior, 10(3), 750-760.

Balconi, M., \& Vanutelli, M. E. (2016a). Competition in the brain. The contribution of EEG and ANIRS modulation and personality effects in social ranking. Frontiers in Psychology. Psychopathology. 13 October 2016.

Balconi, M., \& Vanutelli, M. E. (2016b). Inter-brains cooperation. Hyperscanning and self-perception in joint actions. Journal of Clinical and Experimental Neuropsychology, 39(6), 607-620.

Balconi, M., Crivelli, D. \& Vanutelli, M. E. (2017).Why to cooperate is better than to compete: Brain and personality components. BMC Neuroscience, 18, Article number 68.

Baron-Cohen, S., Ring, H., Moriarty, J., Schmitz, B., Costa, D., \& Ell, P. (1994). Recognition of mental state terms: clinical findings in children with autism and a functional neuroimaging study of normal adults. British Journal of Psychiatry, 165(5), 640-649.

Başar, E. (1999). Brain function and oscillations: integrative brain function neurophysiology and cognitive processes. Berlin: Springer. 
Beck, A. T., Steer, R. A., \& Brown, G. K. (1996). Manual for the Beck Depression Inventory - II. San Antonio: Psychological Corporation.

Billeke, P., Zamorano, F., Cosmelli, D., \& Aboitiz, F. (2013). Oscillatory brain activity correlates with risk perception and predicts social decisions. Cerebral Cortex, 23(12), 2872-2883.

Boone, C., De Brabander, B., \& van Witteloostuijn, A. (1999). The impact of personality on behavior in five Prisoner's Dilemma games. Journal of Economic Psychology, 20(3), 343-377.

Cheung, C. H., Rutherford, H. J., Mayes, L. C., \& McPartland, J. C. (2010). Neural responses to faces reflect social personality traits. Social Neuroscience, 5(4), 351-359.

Chiao, J. Y., Harada, T., Oby, E. R., Li, Z., Parrish, T., \& Bridge, D. J. (2009). Neural representations of social status hierarchy in human inferior parietal cortex. Neuropsychologia, 47(2), 354-363.

Chung, D., Yun, K., \& Jeong, J. (2015). Decoding covert motivations of free riding and cooperation from multi-feature pattern analysis of EEG signals. Social Cognitive and Affective Neuroscience, 10(9), 1210-1218.

Cristofori, I., Moretti, L., Harquel, S., Posada, A., Deiana, G., Isnard, J., ... Sirigu, A. (2013). Theta signal as the neural signature of social exclusion. Cerebral Cortex, 23(10), 2437-2447.

Cui, F., Zhu, X., Duan, F., \& Luo, Y. (2015). Instructions of cooperation and competition influence the neural responses to others' pain: An ERP study. Social Neuroscience, 11(3), 289-296.

Cui, X., Bryant, D. M., \& Reiss, A. L. (2013). NIRS-based hyperscanning reveals increased interpersonal coherence in superior frontal cortex during cooperation. NeuroImage, 59(3), 2430-2437.

Davidson, R. J. (1993). Cerebral asymmetry and emotion: conceptual and methodological conundrums. Cognition \& Emotion, 7(1), 115-138.

De Vico Fallani, F., Nicosia, V., Sinatra, R., Astolfi, L., Cincotti, F., Mattia, D., ... Babiloni, F. (2010). Defecting or not defecting: how to "read" human behavior during cooperative games by EEG measurements. PLoS ONE, 5(12), e14187.

Decety, J., Jackson, P. L., Sommerville, J. A., Chaminade, T., \& Meltzoff, A. N. (2004). The neural bases of cooperation and competition: An fMRI investigation. NeuroImage, 23(2), 744-751.

Dommer, L., Jäger, N., Scholkmann, F., Wolf, M., \& Holper, L. (2012). Betweenbrain coherence during joint $\mathrm{n}$-back task performance: A two-person 
functional near-infrared spectroscopy study. Behavioural Brain Research, 234(2), 212-222.

Fletcher, P. C., Happé, F., Frith, U., Baker, S. C., Dolan, R. J., Frackowiak, R. S. J., \& Frith, C. D. (1995). Other minds in the brain: a functional imaging study of "theory of mind" in story comprehension. Cognition, 57(3), 109-128.

Fukui, H., Murai, T., Shinozaki, J., Aso, T., Fukuyama, H., Hayashi, T., \& Hanakawa, T. (2006). The neural basis of social tactics: an fMRI study. Neuroimage, 32(2), 913-920.

Funane, T., Kiguchi, M., Atsumori, H., Sato, H., Kubota, K., \& Koizumi, H. (2011). Synchronous activity of two people's prefrontal cortices during a cooperative task measured by simultaneous near-infrared spectroscopy. Journal of Biomedical Optics, 16(7), 077011.

Gallagher, H. L., Jack, A. I., Roepstorff, A., \& Frith, C. D. (2002). Imaging the intentional stance in a competitive game. Neuroimage, $16(3 \mathrm{Pt} 1), 814-821$.

Gao, L., Xu, J., Zhang, B., Zhao, L., Harel, A., \& Bentin, S. (2009). Aging effects on early-stage face perception: an ERP study. Psychophysiology, 46(5),

Goel, V., Grafman, J., Sadato, N., \& Hallett, M. (1995). Modeling other minds. Neuroreport, 6(13), 1741-1746.

Goldman, M., Stockbauer, J. W., \& McAuliffe, T. G. (1977). Intergroup and intragroup competition and cooperation. Journal of Experimental Social Psychology, 113(1), 81-88.

Grossmann, T., \& Johnson, M. H. (2010). Selective prefrontal cortex responses to joint attention in early infancy. Biology Letters, 6(4), 540-543.

Grossmann, T., Oberecker, R., Koch, S. P., \& Friederici, A. D. (2010). The developmental origins of voice processing in the human brain. Neuron, 65(6), 852-858.

Harmon-Jones, E., Gable, P. A., \& Peterson, C. K. (2010). The role of asymmetric frontal cortical activity in emotion-related phenomena: a review and update. Biological Psychology, 84(3), 451-462.

Haruno, M., \& Kawato, M. (2009). Activity in the superior temporal sulcus highlights learning competence in an interaction game. The Journal of Neuroscience, 29(14), 4542-4547.

Holper, L., Scholkmann, F., \& Wolf, M. (2012). Between-brain connectivity during imitation measured by fNIRS. NeuroImage, 63(1), 212-222.

Jackson, D. C., Mueller, C. J., Dolski, I., Dalton, K. M., Nitschke, J. B., Urry, H. L., ... Davidson, R. J. (2003). Now you feel it, now you don't: frontal 
brain electrical assymetry and individual differences in emotion regulation. Psychological Science, 14(6), 612-617.

Karafin, M. S., Tranel, D., \& Adolphs, R. (2004). Dominance attributions following damage to the ventromedial prefrontal cortex. Journal of Cognitive Neuroscience, 16(10), 1796-1804.

Kawasaki, M., Yamada, Y., Ushiku, Y., Miyauchi, E., \& Yamaguchi, Y. (2013). Inter-brain synchronization during coordination of speech rhythm in human-to-human social interaction. Scientific Reports, 3, 1-8.

Knyazev, G. G., Slobodskoj-Plusnin, J. Y., \& Bocharov, A. V. (2009). Eventrelated delta and theta synchronization during explicit and implicit emotion processing. Neuroscience, 164(4), 1588-1600.

Konvalinka, I., Bauer, M., Stahlhut, C., Hansen, L. K., Roepstorff, A., \& Frith, C. D. (2014). Frontal alpha oscillations distinguish leaders from followers: Multivariate decoding of mutually interacting brains. NeuroImage, 94, 79-88.

Koslow, K., Mendes, W. B., Pajtas, P. E., \& Pizzagalli, D. A. (2013). Greater left resting intracortical activity as a buffer to social threat. Psychological Science, 22(5), 641-649.

Levitan, R., Hasey, G., \& Sloman, L. (2009). Major depression and the involuntary defeat strategy: biological correlates. In P. Gilbert \& L. Sloman (Eds.), Subordination and defeat: an evolutionary approach tomood disorders and their therapy. (pp. 95-120). Manhaw: Lawrence Erlbaum Associates.

Lindenberger, U., Li, S.-C., Gruber, W., \& Müller, V. (2009). Brains swinging in concert: cortical phase synchronization while playing guitar. $B M C$ Neuroscience, 10(1), 22.

Lloyd-Fox, S., Blasi, A., Volein, A., Everdell, N., Elwell, C. E., \& Johnson, M. H. (2009). Social perception in infancy: a near infrared spectroscopy study. Child Development, 80(4), 986-999.

Ludwig, K. A., Miriani, R. M., Langhals, N. B., Joseph, M. D., Anderson, D. J., \& Kipke, D. R. (2009). Using a common average reference to improve cortical neuron recordings from microelectrode arrays. Journal of Neurophysiology, 101(3), 1679-1689.

Marsh, A. A., Blair, K. S., Jones, M. M., Soliman, N., \& Blair, R. J. R. (2009). Dominance and submission: the ventrolateral prefrontal cortex and responses to status cues, Journal of cognitive neuroscience, 21(4), 713-724.

Minagawa-Kawai, Y., Matsuoka, S., Dan, I., Naoi, N., Nakamura, K., \& Kojima, S. (2009). Prefrontal activation associated with social attachment: facial-emotion recognition in mothers and infants. Cerebral Cortex, 19(2), 284-292. 
Mitchell, J. P., Banaji, M. R., \& Macrae, C. N. (2005). General and specific contributions of the medial prefrontal cortex to knowledge about mental states. Neuroimage, 28(4), 757-762.

Mu, Y., Fan, Y., Mao, L., \& Han, S. (2008). Event-related theta and alpha oscillations mediate empathy for pain. Brain Research, 1234, 128-136.

Naeem, M., Prasad, G., Watson, D. R., \& Kelso, J. A. S. (2012). Electrophysiological signatures of intentional social coordination in the 10-12Hz range. NeuroImage, 59(2), 1795-1803.

Oostenveld, R., \& Praamstra, P. (2001). The five percent electrode system for high-resolution EEG and ERP measurements. Clinical Neurophysiology, $112(4), 713-719$.

Paré, D. (2003). Role of the basolateral amygdala in memory consolidation. Progress in Neurobiology, 70(5), 409-420.

Saito, D. N., Tanabe, H. C., Izuma, K., Hayashi, M. J., Morito, Y., Komeda, H., ... Sadato, N. (2010). "Stay tuned": inter-individual neural synchronization during mutual gaze and joint attention. Frontiers in Integrative Neuroscience, 4, 127.

Sänger, J., Müller, V., \& Lindenberger, U. (2012). Intra- and interbrain synchronization and network properties when playing guitar in duets. Frontiers in Human Neuroscience, 6, 312.

Schippers, M. B., Roebroeck, A., Renken, R., Nanetti, L., \& Keysers, C. (2010). Mapping the information flow from one brain to another during gestural communication. Proceedings of the National Academy of Sciences of the United States of America, 17(20), 9388-9393.

Spielberger, C. D., Gorsuch, R. L., Lushene, R. E., Vagg, P. R., \& Jacobs, G. A. (1970). STAI Manual for the State-Trait Anxiety Inventory. Palo Alto: Consulting Psychologists Press.

Stephens, G. J., Silbert, L. J., \& Hasson, U. (2010). Speaker-listener neural coupling underlies successful communication. Proceedings of the National Academy of Sciences of the United States of America, 107(32), 14425-30.

Suda, M., Takei, Y., Aoyama, Y., Narita, K., Sato, T., Fukuda, M., \& Mikuni, M. (2010). Frontopolar activation during face-to-face conversation: An in situ study using near-infrared spectroscopy. Neuropsychologia, 48(2), 441-447.

Suzuki, S., Niki, K., Fujisaki, S., \& Akiyama, E. (2011). Neural basis of conditional cooperation. Social Cognitive and Affective Neuroscience, 6(3), $338-347$. 
Tauer, J. M., \& Harackiewicz, J. M. (2004). The effects of cooperation and competition on intrinsic motivation and performance. Journal of Personality and Social Psychology, 86(6), 849-861.

Tognoli, E., Lagarde, J., DeGuzman, G. C., \& Kelso, J. A. (2007). The phi complex as a neuromarker of human social coordination. Proceedings of the National Academy of Sciences of the United States of America, 104, 8190-8195.

Urry, H. L., Nitschke, J. B., Dolski, I., Jackson, D. C., Dalton, K. M., Mueller, C. J., ... Davidson, R. J. (2004). Making a life worth living. Neural correlates of well-being. Psychological Science, 15(6), 367-372.

Vanutelli, M. E., \& Balconi, M. (2015). Perceiving emotions in human-human and human-animal interactions: Hemodynamic prefrontal activity (fNIRS) and empathic concern. Neuroscience Letters, 605, 1-6.

Vanutelli, M. E., Nandrino, J.-L., \& Balconi, M. (2016). The boundaries of cooperation: sharing and coupling from ethology to neuroscience. Neuropsychological Trends, (19), 83-104.

Weiland, S., Hewig, J., Hecht, H., Mussel, P., \& Miltner, W. H. (2012). Neural correlates of fair behavior in interpersonal bargaining. Social Neuroscience, 7(5), 537-551.

Yun, K., Watanabe, K., \& Shimojo, S. (2012). Interpersonal body and neural synchronization as a marker of implicit social interaction. Scientific Reports, 2, 959.

Zink, C. F., Tong, Y., Chen, Q., Bassett, D. S., Stein, J. L., \& Meyer-lindenberg, A. (2008). Know your place: Neural Processing of Social Hierarchy in Humans. Neuron, 58(2), 273-283. 
